\title{
Short-term results of percutaneous closure of patent foramen ovale guided by transoesophageal echocardiography in patients with cryptogenic stroke: A retrospective study
}

\author{
Yilong Guo ${ }^{1}$, Zhensu $\mathrm{Shi}^{2}$, Yin Zheng ${ }^{3}$, Caichan $\mathrm{Xie}^{2}$, Jiao $\mathrm{Yi}^{2}$, Zelun $\mathrm{Chen}^{2}$, Yue Shu ${ }^{2}$, \\ and Dexing Zhou ${ }^{2}$ \\ ${ }^{1}$ Medical School of Chinese PLA Beijing People's Republic of China \\ ${ }^{2}$ Department of Cardiovascular Surgery The Second Affiliated Hospital of Hainan Medical \\ University Haikou Hainan People's Republic of China \\ ${ }^{3}$ Department of Special Medical Services Hainan Cancer Hospital Haikou Hainan People's \\ Republic of China
}

December 31, 2021

\begin{abstract}
Background: This study aimed to assess the short-term (12 months) results of PFO occlusion guided by transoesophageal echocardiography (TEE) and the results of regular transthoracic ultrasound foaming test (UFT). Methods: Data of 75 patients who underwent interventional therapy for PFO and CS were retrospectively analysed. The patients were grouped according to their preoperative UFT results: group A, small volume of right-to-left shunts; group B, moderate volume of right-to-left shunts; and group C, large volume of right-to-left shunts. All patients were treated with an Amplatzer occluder under TEE guidance. UFT follow-up was conducted regularly until 12 months after surgery. Results: No remarkable differences in preoperative data, length of hospital stay, or operative time were noted between the groups. Length of the PFO and diameter of the occluder differed between the groups: group $\mathrm{A}=$ group $\mathrm{Bp}<0.001)$. One patient in group $\mathrm{C}$ developed recurrent stroke 11 months postoperatively. Two patients in group $\mathrm{C}$ developed atrial arrhythmia, which improved after 3 months of antiarrhythmic treatment. However, 19 patients still had positive UFT results 12 months postoperatively. Furthermore, the positive UFT rate 12 months postoperatively differed between the groups: group $\mathrm{A}=$ group $\mathrm{Bp}<0.05)$. Conclusions: In patients with $\mathrm{PFO}$ and CS, interventional therapy guided by TEE could lead to satisfactory short-term (12 months) outcomes. A longer PFO and preoperative large-volume shunt were negatively associated with a negative UFT rate 12 months postoperatively. Further studies are required to clarify the relationship between positive UFT results postoperatively and stroke recurrence.
\end{abstract}

\section{INTRODUCTION}

The incidence of ischaemic stroke in China has been increasing annually. Despite extensive examination, the aetiology is still unclear in approximately $40 \%$ of patients with stroke, and this is referred to as cryptogenic stroke (CS). ${ }^{1,2}$ Studies have shown that a patent foramen ovale (PFO) is an independent risk factor for CS, especially in patients $<60$ years of age with atrial septal aneurysm or a large volume of right-to-left shunts. ${ }^{3,4}$ Compared with pharmacological treatments, PFO interventional therapy can significantly reduce the recurrence rate of stroke ${ }^{5,6}$; therefore, methods of diagnosis and interventional therapies for PFO have been rapidly developed. The transthoracic ultrasound foaming test (UFT) is an important examination that significantly improves the detection rate of $\mathrm{PFO}^{7}$; however, most $\mathrm{PFO}$ interventional therapies are currently performed using radiography, which is associated with the risks of exposure to radiation. Furthermore, followup data on postoperative transthoracic UFT are lacking. ${ }^{4}{ }^{6}$ Hence, this study was designed to assess the 
short-term (12 months) effects of PFO interventional therapy guided by transoesophageal echocardiography (TEE) and the results of regular transthoracic UFTs after surgery.

\section{METHODS}

\section{Patients}

This retrospective study included data of 78 patients who underwent PFO interventional therapy from April 2019 to July 2020. The inclusion criteria were as follows: 1) a clear diagnosis of CS and PFO; 2) age $<60$ years; and 3) transthoracic UFT performed strictly according to requirements (before surgery and at $3,6,9$, and 12 months postoperatively). The exclusion criteria were 1) severe organ dysfunction; 2) atrial fibrillation or atrial flutter diagnosed before surgery; or 3) other heart diseases requiring simultaneous surgical treatment. Of these 78 patients, 2 were excluded due to preoperative atrial fibrillation and 1 was excluded due to severe liver disease. After applying the exclusion criteria, 75 patients were finally included. This study was approved by the Hainan Medical University Clinic Institutional Review Board. Due to the retrospective design of this study, the need for obtaining patient informed consent was waived.

\section{Transthoracic UFT}

First, activated normal saline was prepared using two 10-mL syringes: the first syringe was used to draw $8 \mathrm{~mL}$ of normal saline and the other contained $1 \mathrm{~mL}$ of air and $1 \mathrm{~mL}$ of the patient's blood. The syringes were connected to a three-way valve and their contents were rapidly injected back and forth to fully mix the blood, normal saline, and air to obtain $10 \mathrm{~mL}$ of activated normal saline. ${ }^{8,9}$ Two syringes, each containing $10 \mathrm{~mL}$ of activated normal saline, were prepared for each patient.

Second, while the patient was in the supine position, an apical four-chamber view was obtained using transthoracic echocardiography. One syringe of activated normal saline was rapidly injected through the cubital vein of the patient. If a microbubble entered the left atrium within 10 cardiac cycles, it was recognised as a positive result. Afterwards, the patients were asked to perform the Valsalva manoeuvre (thoracic pressure [?]40 $\mathrm{mmHg}$ ) for more than $10 \mathrm{~s}$. The other $10 \mathrm{~mL}$ of activated normal saline was rapidly injected through the cubital vein. ${ }^{10}$ The number of microbubbles in the left atrium during three cardiac cycles was recorded, and the partial flow of PFO was graded as follows: no shunt, no microbubbles in the left atrium; small, 1-10 microbubbles in the left atrium; moderate, 11-25 microbubbles in the left atrium; and large, $>25$ microbubbles in the left atrium. ${ }^{8,9}$

Third, the patients were divided into three groups according to their transthoracic UFT results: group A, small-volume shunt group; group B, moderate-volume shunt group; and group C, large-volume shunt group.

\section{Surgical methods}

All surgeries were performed under general anaesthesia and guided by TEE. The length of the PFO was measured using TEE in the double vena cava section of the middle oesophagus, and an occluder was selected based on the measured PFO length. The right femoral vein was punctured, and the interventional track was established: right femoral vein - right atrium - PFO - left atrium. An Amplatzer occluder (Abbott Medical, Nathan Lane North Plymouth, MN, USA) was implanted along this track. After the occluder was released, the interventional catheter was removed, and pressure was applied over the right femoral vein puncture site to secure haemostasis.

\section{Follow-up}

All patients were regularly treated with aspirin for 6 months (3-5 mg/kg/day). At 3, 6, 9, and 12 months postoperatively, all the patients returned to the hospital for transthoracic echocardiography, transthoracic UFT, chest radiography, and electrocardiography. All echocardiography and UFT results were evaluated by two doctors independently. If the reports of these doctors were inconsistent, the opinion of a third doctor was requested, and the opinion of the majority was the final data included in the analysis.

\section{Data collection and processing}


Preoperative data included the sex ratio, age, weight, height, comorbidities, right ventricular diameter (RVD), left atrial diameter (LAD), main pulmonary artery diameter (PAD), pulmonary systolic pressure (PSP), pulmonary vascular resistance (PVR), left ventricular end-diastolic diameter (LVEDD), and left ventricular ejection fraction (LVEF).

Intraoperative data collected included the length of the PFO, operative time, and diameter of the occluder. Follow-up data collected included common postoperative complications (pericardial tamponade, occluder migration, and local vascular injury), length of hospital stay, stroke recurrence, atrial fibrillation, atrial flutter, and UFT results.

\section{Statistical analysis}

Continuous data are expressed as mean \pm standard deviation (SD), while categorical variables are expressed as percentages. Between-group comparisons were performed using the one-way analysis of variance, chi-square test, or Fisher's exact test, as appropriate.

Kaplan-Meier analysis was used to analyse the relationship between UFT results and follow-up time, and Cox regression analysis was used to analyse the effects of various factors on the postoperative UFT results. All statistical data were processed with SPSS version 19.0 (IBM SPSS, Armonk, NY, USA), and $p$ values $<0.05$ were considered reflective of statistical significance.

\section{RESULTS}

\section{Preoperative information}

Group A included 21 patients, with a mean age of 45.05 \pm 8.992 years; group B included 22 patients, with a mean age of $45.09 \pm 9.314$ years; and group $\mathrm{C}$ included 32 patients, with a mean age of $41.25 \pm 10.352$ years. There were no significant differences in preoperative data between the groups. The clinical and echocardiographic characteristics of the study population are presented in Tables 1 and 2, respectively.

Table 1 Clinical characteristics of the study population

\begin{tabular}{lllll}
\hline & Group A $(\mathbf{n = 2 1})$ & Group B $(\mathbf{n = 2 2})$ & Group C $(\mathbf{n = 3 2})$ & $p$ value \\
\hline Female sex & $14(66.67 \%)$ & $11(50.00 \%)$ & $12(37.50 \%)$ & 0.119 \\
Age $($ mean \pm SD, years $)$ & $45.05 \pm 8.992$ & $45.09 \pm 9.314$ & $41.25 \pm 10.352$ & 0.247 \\
Weight $($ mean \pm SD, $\mathrm{kg})$ & $60.92 \pm 7.855$ & $59.59 \pm 11.548$ & $56.72 \pm 8.938$ & 0.263 \\
Height $($ mean $\pm \mathrm{SD}, \mathrm{cm})$ & $163.05 \pm 7.965$ & $160.68 \pm 7.656$ & $160.06 \pm 5.224$ & 0.287 \\
Diabetes & $2(9.52 \%)$ & $4(18.18 \%)$ & $1(3.13 \%)$ & 0.178 \\
Hypertension & $7(33.33 \%)$ & $8(36.36 \%)$ & $7(21.88 \%)$ & 0.467 \\
Hyperlipidaemia & $1(4.76 \%)$ & $4(18.18 \%)$ & $1(3.13 \%)$ & 0.112 \\
\hline
\end{tabular}

$\mathrm{SD}$, standard deviation

Group A, small-volume shunt group; Group B, moderate-volume shunt group; and Group C, large-volume shunt group

Table 2 Echocardiographic characteristics of the study population

\begin{tabular}{lllll}
\hline & Group A $(\mathbf{n = 2 1})$ & Group B $(\mathbf{n = 2 2})$ & Group C $(\mathbf{n = 3 2})$ & $p$ value \\
\hline RVD (mm) & $23.24 \pm 2.879$ & $22.05 \pm 4.326$ & $21.47 \pm 4.333$ & 0.290 \\
LAD (mm) & $31.48 \pm 5.437$ & $29.45 \pm 3.776$ & $28.69 \pm 5.515$ & 0.147 \\
PAD (mm) & $21.29 \pm 3.036$ & $20.32 \pm 2.255$ & $20.47 \pm 3.233$ & 0.500 \\
LVEDD (mm) & $43.81 \pm 2.839$ & $42.82 \pm 3.187$ & $42.72 \pm 2.667$ & 0.367 \\
LVEF (\%) & $63.00 \pm 6.116$ & $65.50 \pm 2.596$ & $64.98 \pm 5.097$ & 0.205 \\
PSP (mmHg) & $22.62 \pm 4.031$ & $23.09 \pm 3.531$ & $24.44 \pm 2.590$ & 0.121
\end{tabular}




\begin{tabular}{lllll}
\hline & Group A $(\mathbf{n}=\mathbf{2 1})$ & Group B $(\mathbf{n}=\mathbf{2 2})$ & Group C $(\mathbf{n}=\mathbf{3 2})$ & $p$ value \\
\hline $\operatorname{PVR}\left(\mathrm{dyn} \cdot \mathrm{s} \cdot \mathrm{cm}^{-5}\right)$ & $150.10 \pm 26.474$ & $164.55 \pm 20.871$ & $158.47 \pm 24.397$ & 0.149 \\
\hline
\end{tabular}

LAD, left atrial diameter; LVEDD, left ventricular end-diastolic diameter; LVEF, left ventricular ejection fraction; PAD, main pulmonary artery diameter; PSP, pulmonary systolic pressure; PVR, pulmonary vascular resistance; RVD, right ventricular diameter.

Group A, small-volume shunt group; Group B, moderate-volume shunt group; and Group C, large-volume shunt group

\section{Perioperative data}

All patients were successfully treated with PFO interventional therapy, and there was no incidence of perioperative pericardial tamponade, occluder migration, cardiac rupture, or malignant arrhythmia. No significant difference was found in the operative time and length of stay between the groups $(p>0.05)$. The length of PFO differed between the groups as follows: group $\mathrm{A}=$ group $\mathrm{B}<$ group $\mathrm{C}(p<0.001)$. Patients in group $\mathrm{C}$ had significantly larger occluders than patients in the other two groups $(p<0.001)$. The perioperative data of the study population are presented in Table 3.

Table 3 Perioperative data of the study population

\begin{tabular}{lllll}
\hline & Group A $(\mathbf{n = 2 1})$ & Group $(\mathbf{n = 2 2})$ & Group C $(\mathbf{n = 3 2})$ & $p$ value \\
\hline Time (min) & $44.71 \pm 25.714$ & $35.64 \pm 20.984$ & $38.50 \pm 25.821$ & 0.464 \\
Length of PFO (mm) & $9.03 \pm 4.616^{\mathrm{a}}$ & $10.55 \pm 3.546^{\mathrm{b}}$ & $14.021 \pm 3.047^{\mathrm{a}, \mathrm{b}}$ & $<0.001$ \\
Occluder (mm) & $19.67 \pm 3.055^{\mathrm{a}}$ & $19.91 \pm 3.191^{\mathrm{b}}$ & $23.25 \pm 3.800^{\mathrm{a}, \mathrm{b}}$ & $<0.001$ \\
Length of stay (days) & $12.24 \pm 6.480$ & $13.50 \pm 8.478$ & $13.00 \pm 8.658$ & 0.875 \\
\hline
\end{tabular}

Statistical comparisons: comparison between groups, ${ }^{\mathrm{a}} p<0.01 ;{ }^{\mathrm{b}} p<0.05$

$\mathrm{PFO}$, patent foramen ovale

Group A, small-volume shunt group; Group B, moderate-volume shunt group; and Group C, large-volume shunt group

\section{Follow-up data}

All patients in this study were followed up regularly for 12 months. There was no new migraine, pericardial tamponade, occluder migration, occluder-related valve dysfunction, death, bleeding, or oesophageal perforation within 12 months postoperatively. Only one 38-year-old female patient in group $\mathrm{C}$ developed transient recurrent stroke 11 months postoperatively. She was discharged without any sequelae after 7 days of treatment; she still had a positive UFT result 12 months postoperatively. There was no arrhythmia in groups A and B. Two patients in group $\mathrm{C}$ developed atrial arrhythmias (alternating atrial fibrillation and atrial flutter) 3 months postoperatively. They were treated with amiodarone $(200 \mathrm{mg} /$ day $)$ and recovered after 3 months.

All patients underwent UFT regularly after surgery. The residual shunt on the atrial septum was excluded using transthoracic echocardiography before UFT (Fig. 1). Twelve months after surgery, no patients in group A had a positive UFT (Fig. 2). Three patients in group B had positive UFT results (Fig. 3), whereas 16 patients in group $\mathrm{C}$ still had positive UFT results (Fig. 3). The number of patients who had positive UFT results 12 months postoperatively differed between the groups as follows: group $\mathrm{A}=$ group $\mathrm{B}<$ group $\mathrm{C}$ $(p<0.05)$. The positive UFT results during follow-up are presented in Table 4.

Table 4 Positive results of UFT during follow-up 


\begin{tabular}{llll}
\hline & Group A $(\mathbf{n = 2 1})$ & Group B $(\mathbf{n = 2 2 )}$ & Group C (n=32) \\
\hline Three months & $17(80.95 \%)$ & $21(95.45 \%)$ & $32(100 \%)$ \\
Six months & $8(38.10 \%)$ & $13(59.09 \%)$ & $31(96.88 \%)$ \\
Nine months & $2(9.52 \%)$ & $7(31.82 \%)$ & $23(71.88 \%)$ \\
Twelve months & $0(0)$ & $3(13.64 \%)$ & $16(50.00 \%)$ \\
\hline
\end{tabular}

UFT, ultrasound foaming test

Group A, small-volume shunt group; Group B, moderate-volume shunt group; and Group C, large-volume shunt group

Kaplan-Meier analysis showed that the positive UFT rate gradually decreased during follow-up (Fig. 4). Cox regression analysis showed that the positive UFT rate in group C was higher than that in group A $(p$ $=0.002)$ (Fig. 5). There was no significant difference in the positive UFT rate between groups A and B $(p$ $=0.135$ ). Moreover, a preoperative large-volume shunt was negatively associated with a negative UFT rate 12 months postoperatively ( $R R=0.255, p=0.003)$. Age, sex, weight, height, RV, PA, PSP, PVR, LVEDD, LVEF, and LA had no significant effect on postoperative UFT results $(p>0.05)$.

\section{DISCUSSION}

The foramen ovale is an important structure in the foetal heart that allows oxygenated blood to flow from the right atrium into the left atrium and ventricle, which then supplies blood and oxygen to the whole body. ${ }^{11}$ After birth, with a decrease in pulmonary artery pressure and an increase in left atrial pressure, the foramen ovale closes spontaneously in approximately $75 \%$ of the population. ${ }^{12}$ In others, the foramen ovale does not close, resulting in a PFO. ${ }^{13,}{ }^{14}$ Studies have shown that a PFO is a risk factor for CS; hence, studies on interventional therapy for PFO have been increasing for decades. ${ }^{15}$

Initially, PFO interventional therapies were guided by X-ray imaging. However, its development was limited owing to the use of contrast agents and exposure to X-rays. Therefore, surgeons have recently begun to perform PFO interventional closure under the guidance of TEE. ${ }^{16,17}$ In our study, all patients were successfully treated with interventional therapy under TEE guidance, with no pericardial tamponade, death, or other major complications. The most common complications after PFO interventional therapy are recurrent stroke and atrial fibrillation ${ }^{18,19}$; however, in this study, only one patient developed transient recurrent stroke 11 months postoperatively, and two patients developed atrial arrhythmias 3 months postoperatively. Together, these results suggest that PFO interventional therapy guided by TEE could lead to satisfactory short-term (12 months) results, which is consistent with the findings of previous studies. ${ }^{17}$

Herein, the positive UFT rate gradually decreased. However, there were still 19 patients, most of whom belonged to group C, with positive UFT results 12 months postoperatively; this was the most important finding of this study. Previous studies have shown that the recurrence rate of stroke after PFO occlusion in patients with CS is $2.0 \%,{ }^{19}$ the reason for which is still unclear. In our study, one patient in group C developed transient recurrent stroke 11 months postoperatively and she still had a positive UFT result 12 months postoperatively. A positive UFT result after surgery means that microthrombi and microbubbles can still be shunted from the right atrium to left atrium through the PFO occluder. As the number of patients who developed recurrent stroke postoperatively in this study was small, further statistical analysis could not be conducted. Thus, we cannot rule out the possibility that a persistent positive UFT result after surgery is a risk factor for recurrent stroke. After detailed analysis, there were two possible reasons for why 16 patients in group C still had positive UFT results 12 months postoperatively. First, the Amplatzer occluder, which is used worldwide, was used in this study. It has a metal-braided mesh structure without a film on the surface. Therefore, it cannot prevent the passage of microthrombi and microbubbles. However, the occluder is gradually embedded in the intima after it is released. This implies that when the occluder is completely embedded, microthrombi and microbubbles would be unable to pass through it, resulting in a negative UFT result. ${ }^{19,20}$ The present study shows that there are individual differences in the process 
by which the occluder is embedded. Moreover, patients with a large volume of right-to-left shunts prior to surgery will have a longer embedding process. Second, in this study, the length of the PFO and the diameter of the occluder in group $\mathrm{C}$ were larger than those in group A and group B, while the positive UFT rate 12 months postoperatively in group $\mathrm{C}$ was higher than that in group $\mathrm{A}$ and group $\mathrm{B}$. Furthermore, the diameter of the occluder used in this study was equal to the length of the PFO $+10 \mathrm{~mm}$. Considering all these findings, it could be concluded that with an increase in the PFO length, the diameter of the occluder increases and the embedding process is prolonged; hence, UFT positivity after surgery is observed in the long term.

This study has some limitations as it was a retrospective, non-randomised study; therefore, some selection bias exists. Further prospective, randomised, large-scale, and long-term studies are required to clarify the changes in UFTs after PFO occlusion and to verify the relationship between positive UFT results postoperatively and stroke recurrence.

\section{CONCLUSIONS}

In patients with PFO and CS, interventional therapy guided by TEE could lead to satisfactory shortterm (12 months) outcomes. Although the positive UFT rate gradually decreased, some patients still had positive UFT results 12 months postoperatively. A large volume of right-to-left shunts and a longer PFO preoperatively were risk factors for positive UFT results postoperatively. Further studies are required to clarify the relationship between positive UFT results postoperatively and stroke recurrence.

\section{DECLARATIONS}

\section{Ethics approval}

This study was approved by the Hainan Medical University Clinic Institutional Review Board. The protocol of this study was performed in accordance with the Declaration of Helsinki.

\section{Patient consent statement}

The need for patient consent was waived due to the retrospective study design.

\section{Consent for publication}

Not applicable.

\section{Availability of data and materials}

All data generated or analysed during this study are included in this published article.

\section{Conflict of interest disclosure}

The authors declare that they have no competing interests.

\section{Author contributions}

YG and ZS conceptualised and designed the study. YS and DZ provided administrative support. YZ and JY provided study materials or helped in recruiting patients. ZC and YS collected and assembled all data. CX and DZ analysed and interpreted the data. YG and ZS wrote the manuscript. All authors provided their final approval of the manuscript.

\section{Authors' information}

Yilong Guo: 847287951@qq.com.

Zhensu Shi: keroroooo@163.com.

Yin Zheng: 2359187714@qq.com.

Caichan Xie: xcc201904@163.com. 
Jiao Yi: yj201904@163.com.

Zelun Chen: 524978035@qq.com.

Yue Shu: gyl19861027@126.com.

Dexing Zhou: 275183069@qq.com.

\section{Acknowledgements}

Not applicable.

\section{REFERENCES}

1. Steiner MM, Di Tullio MR, Rundek T, et al. Patent foramen ovale size and embolic brain imaging findings among patients with ischemic stroke. Stroke 1998;29:944-948.

2. Hart RG, Miller VT. Cerebral infarction in young adults: a practical approach. Stroke 1983;14:110-114.

3. Mas JL, Derumeaux G, Guillon B, et al. Patent foramen ovale closure or anticoagulation vs. antiplatelets after stroke. N Engl J Med 2017;377:1011-1021.

4. Katsanos AH, Spence JD, Bogiatzi C, et al. Recurrent stroke and patent foramen ovale: a systematic review and meta-analysis. Stroke 2014;45:3352-3359.

5. Kent DM, Dahabreh IJ, Ruthazer R, et al. Anticoagulant vs. antiplatelet therapy in patients with cryptogenic stroke and patent foramen ovale: an individual participant data meta-analysis. Eur Heart J 2015;36:2381-2389.

6. Elmariah S, Furlan AJ, Reisman M, et al. Predictors of recurrent events in patients with cryptogenic stroke and patent foramen ovale within the CLOSURE I (Evaluation of the STARFlex septal closure system in patients with a stroke and/or transient ischemic attack due to presumed paradoxical embolism through a patent foramen ovale) trial. JACC Cardiovasc Interv 2014;7:913-920.

7. Katsanos AH, Psaltopoulou T, Sergentanis TN, et al. Transcranial Doppler versus transthoracic echocardiography for the detection of patent foramen ovale in patients with cryptogenic cerebral ischemia: a systematic review and diagnostic test accuracy meta-analysis. Ann Neurol 2016;79:625-635.

8. Mojadidi MK, Winoker JS, Roberts SC, et al. Accuracy of conventional transthoracic echocardiography for the diagnosis of intracardiac right-to-left shunt: a meta-analysis of prospective studies. Echocardiography 2014;31:1036-1048.

9. Mojadidi MK, Winoker JS, Roberts SC, Msaouel P, Gevorgyan R, Zolty R. Two-dimensional echocardiography using second harmonic imaging for the diagnosis of intracardiac right-to-left shunt: a meta-analysis of prospective studies. Int J Cardiovasc Imaging 2014;30:911-923.

10. Guo YZ, Gao YS, Guo ZN, Niu PP, Yang Y, Xing YQ. Comparison of different methods of valsalva maneuver for right-to-left shunt detection by contrast-enhanced transcranial Doppler. Ultrasound Med Biol 2016;42:1124-1129.

11. Dattilo PB, Kim MS, Carroll JD. Patent foramen ovale. Cardiol Clin 2013;31:401-415.

12. Homma S, Sacco RL. Patent foramen ovale and stroke. Circulation 2005;112:1063-1072.

13. Di Tullio MR. Patent foramen ovale: echocardiographic detection and clinical relevance in stroke. J Am Soc Echocardiogr 2010;23:144-155.

14. Sun YP, Homma S. Patent foramen ovale and stroke. Circ J 2016;80:1665-1673.

15. Bridges ND, Hellenbrand W, Latson L, Filiano J, Newburger JW, Lock JE. Transcatheter closure of patent foramen ovale after presumed paradoxical embolism. Circulation 1992;86:1902-1908. 
16. Akagi T. Transcatheter closure of patent foramen ovale: current evidence and future perspectives. J Cardiol 2021;77:3-9.

17. Han Y, Zhang X, Zhang F. Patent foramen ovale closure by using transesophageal echocardiography for cryptogenic stroke: single center experience in 132 consecutive patients. J Cardiothorac Surg 2020;15:11.

18. Teshome MK, Najib K, Nwagbara CC, Akinseye OA, Ibebuogu UN. Patent foramen ovale: a comprehensive review. Curr Probl Cardiol 2020;45:100392.

19. Mojadidi MK, Zaman MO, Elgendy IY, et al. Cryptogenic stroke and patent foramen ovale. J Am Coll Cardiol 2018;71:1035-1043.

20. Carroll JD, Saver JL, Thaler DE, et al. Closure of patent foramen ovale versus medical therapy after cryptogenic stroke. N Eng J Med 2013;368:1092-1100.

\section{FIGURE LEGENDS}

Fig. 1 Transthoracic echocardiography shows blood flow after PFO occlusion. No residual shunt is noted between the LA and RA. LA, left atrial; LV, left ventricular; PFO, patent foramen ovale; RA, right atrial; $\mathrm{RV}$, right ventricular

Fig. 2 Negative transthoracic UFT result after PFO occlusion. There is no microbubble in the LA. LA, left atrial; LV, left ventricular; PFO, patent foramen ovale; RA, right atrial; UFT, ultrasound foaming test

Fig. 3 Positive transthoracic UFT result after PFO occlusion. There are more than 25 microbubbles in the LA and LV. LA, left atrial; LV, left ventricular; PFO, patent foramen ovale; RA, right atrial; UFT, ultrasound foaming test

Fig. 4 Life-table analysis: the cumulative positive UFT rate for all patients. UFT, ultrasound foaming test

Fig. 5 Cox regression analysis: the cumulative positive UFT rate for different groups. UFT, ultrasound foaming test 
Figure 1

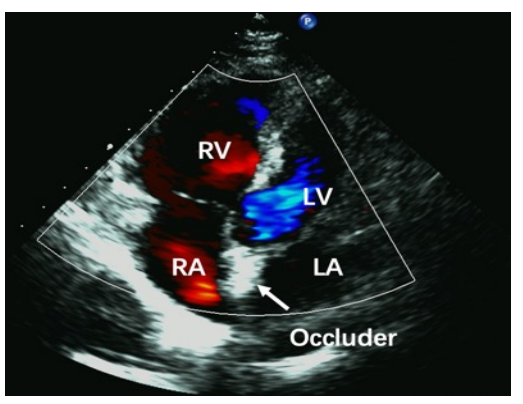

Fig. 1 Transthoracic echocardiography shows blood flow after PFO occlusion. No residual shunt is noted between

the LA and RA. LA, left atrial; LV, left ventricular; PFO, patent foramen ovale; RA, right atrial; RV, right

ventricular 
Figure 2

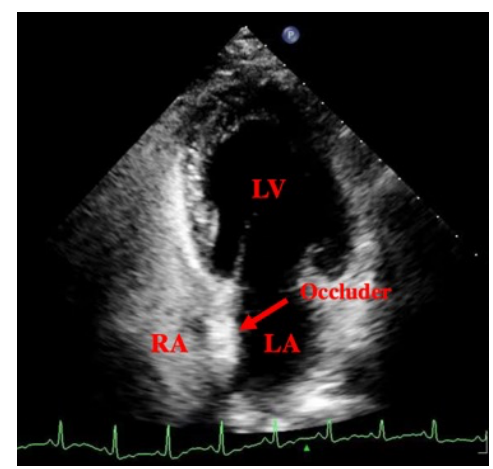

Fig. 2 Negative transthoracic UFT result after PFO occlusion. There is no microbubble in the LA. LA, left atrial;

$\mathrm{LV}$, left ventricular; PFO, patent foramen ovale; RA, right atrial; UFT, ultrasound foaming test 
Figure 3

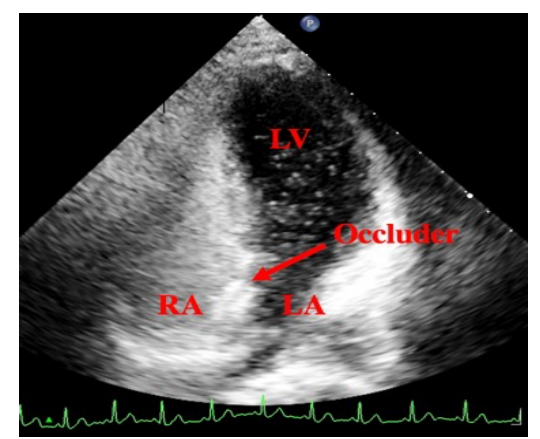

Fig. 3 Positive transthoracic UFT result after PFO occlusion. There are more than 25 microbubbles in the LA and LV. LA, left atrial; LV, left ventricular; PFO, patent foramen ovale; RA, right atrial; UFT, ultrasound foaming test 
Figure 4

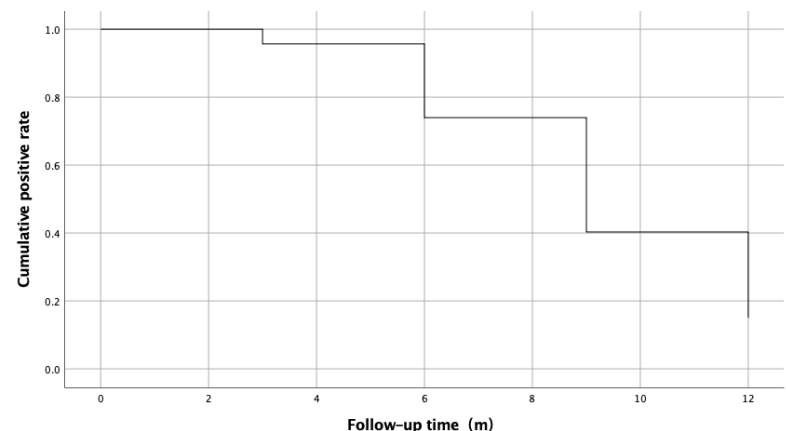

Fig. 4 Life-table analysis: the cumulative positive UFT rate for all patients. UFT, ultrasound foaming test 
Figure 5

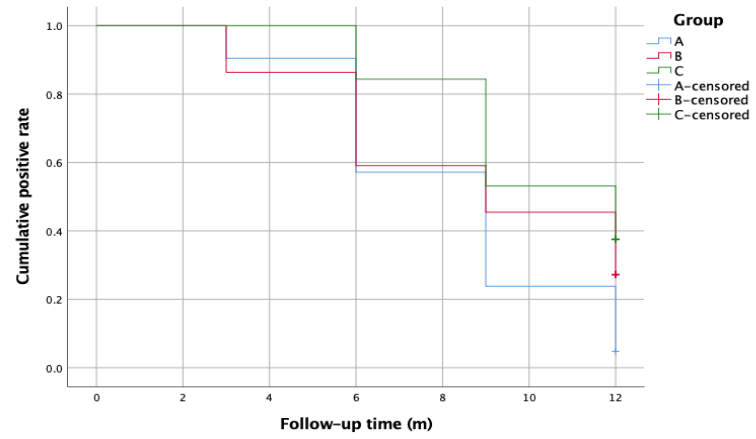

Fig. 5 Cox regression analysis: the cumulative positive UFT rate for different groups. UFT, ultrasound foaming test 